\title{
"Burying" covered coronary stents under drug-eluting stents: A novel approach to ensure long-term stent patency
}

\author{
Matthias Bossard®, Giacomo Maria Cioffi, Mustafa Yildirim, Federico Moccetti, \\ Mathias Wolfrum, Adrian Attinger, Stefan Toggweiler, Richard Kobza, Florim Cuculi \\ Cardiology Division, Heart Center, Luzerner Kantonsspital, Luzern, Switzerland
}

\begin{abstract}
Background: Covered coronary stent (CS) implantation is associated with a high risk for in-stent restenosis (ISR) and stent thrombosis (ST). We describe the outcomes after overstenting ("burying") $C S$ using contemporary drug-eluting stents (DES).

Methods: We analyzed short- and long-term outcomes of consecutive patients who had had a CS implanted, which was consecutively covered ("buried") with a third-generation DES. CSs were primarily post-dilated and then covered with a longer DES overlapping the proximal and distal edges of the CS. To ensure optimal stent expansion and appositions, all lesions were post-dilated using adequately sized non-compliant balloons.

Results: Between 2015 and 2020, 23 patients (mean age $67 \pm 14$ years, $74 \%$ males) were treated using this novel approach. Reasons for implanting CS included treatment of coronary aneurysms $(n=7$; $30 \%)$, coronary perforations ( $n=13 ; 57 \%)$, and aorto-ostial dissections $(n=3 ; 13 \%)$. All CSs were successfully deployed, and no peri-procedural complications occurred. The median time of follow-up was 24.5 (interquartile range [IQR] 11.7-37.9) months. All patients had a 1-month follow-up (FU) and 19/23 (83\%) patients had 12-month FU (FU range 1-60 months). No probable or definite STs occurred, and no cardiovascular deaths were observed. Among patients undergoing angiographic FU (11/23 [48\%]), 1/23 showed angiographically significant ISR 6 months post CS implantation.

Conclusions: Burying a coronary CS under a DES appears to be a safe and promising strategy to overcome the limitations of the currently available CS devices, including a relatively high risk for target lesion failure due to ISR and ST. (Cardiol J 2023; 30, 2: 196-203)
\end{abstract}

Key words: stents, covered stent, aneurysm, perforation, target lesion failure, in-stent restenosis, percutaneous coronary intervention

\section{Introduction}

Coronary covered stents (CS) — stent devices covered with synthetic or biological membranes are generally used to seal coronary perforations [1] or exclude coronary aneurysms [2]. While they are potentially lifesaving in the acute setting, high rates of in-stent-restenosis (ISR) and stent thrombosis (ST) have been observed during mid- and long-term follow-up [3-7]. Indeed, a recent multicenter registry analysis indicated target lesion revascularization (TLR) and ST rates of 5\% and $3 \%$, respectively, after a mean follow-up of $22 \pm 16$ months [7].

The vascular healing of the CS' synthetic material represents a complex process, and the contact of the surface of the implanted stent graft material with extracellular matrix, blood cells, and endothelial

Address for correspondence: Dr. Florim Cuculi, MD, Co-Chief Cardiology Division, Heart Center - Luzerner Kantonsspital, Spitalstrasse 16, 6000 Luzern, Switzerland, tel: +41(0)4120521 34, e-mail: florim.cuculi@luks.ch

Received: 1.01.2021 Accepted: 6.06.2021 Early publication date: 17.08.2021

This article is available in open access under Creative Common Attribution-Non-Commercial-No Derivatives 4.0 International (CC BY-NC-ND 4.0) license, allowing to download articles and share them with others as long as they credit the authors and the publisher, but without permission to change them in any way or use them commercially. 
cells triggers pro-thrombotic processes [8]. Additionally, intimal hyperplasia is uncontrolled and leads to ISR [9]. In contrast, the latest generation of drug-eluting stents (DES) and their antiproliferative properties have tremendously improved angiographic and clinical outcomes of patients undergoing percutaneous coronary intervention (PCI) with stent implantation. Those advancements are attributable to thinner metallic stent platforms, novel biocompatible polymer technologies, and potent antiproliferative drugs, which improve reendothelialization and therefore mitigate the risk for target lesion failure [10, 11].

In this context, combining contemporary CS with novel DES, by over-stenting ("burying") the covered stent under the DES, might help to overcome the limitations related to CS and achieve better short- and long-term outcomes. We describe short- and long-term results of this novel approach of covering coronary CS with the latest generation (thin-strut) DES.

\section{Methods}

\section{Study design}

This analysis derives from the ongoing retrospective L-CAD registry, which was established to assess procedural characteristics and outcomes of patients requiring PCI for complex coronary lesions. This registry complies with the Declaration of Helsinki and was approved by the regional ethics committee (BASEC ID 2019-01067). Patients provided written informed consent for data collection.

We analyzed consecutive patients with coronary aneurysms or coronary perforations, who were treated with the novel approach of combining CS and DES between February 2015 and March 2020. All patients were treated for acute or chronic coronary syndromes at the Cardiology Division of the Luzerner Kantonsspital (Lucerne, Switzerland), a tertiary cardiology facility in Switzerland, which performs annually more than 1600 PCIs.

\section{Percutaneous coronary intervention}

All patients were treated according to our local PCI protocol using a radial or femoral approach. We administered unfractionated heparin in a weight-adapted dosage $(70 \mathrm{U} / \mathrm{kg})$ and ensured that all patients received guideline-recommended dual antiplatelet therapy including acetylsalicylic acid (ASA) plus a loading dose of clopidogrel, ticagrelor, or prasugrel for 12 months. In patients who required anticoagulation, we administered periprocedural ASA and prescribed a novel oral anticoagulant in combination with clopidogrel for 12 months. The patients were otherwise treated according to guideline recommendations for acute or chronic coronary syndromes.

The PCI procedures were performed according to the involved physician's preferences. Of note, our institution routinely uses non-compliant balloons at high pressure ( $>18 \mathrm{~atm})$ for pre-dilatation. For heavily calcified lesions, dedicated tools including rotational atherectomy, scoring balloons, and/ or ultra-high-pressure non-compliant balloons $\left(\mathrm{OPN}^{\mathrm{TM}}\right.$, SIS Medical, Frauenfeld, Switzerland) were used.

Patients with coronary perforations were admitted to our recovery area or cardiac care unit for surveillance and received serial follow-up echocardiograms.

\section{Coronary covered stent devices}

The choice of CS device used for the treatment of a coronary perforation or aneurysm was left to the involved physician's discretion. We implanted either polytetrafluoroethylene or polyurethane CSs. Expanded polytetrafluoroethylene (ePTFE) is a synthetic fluorocarbon polymer with a broad range of applications in medicine, including the manufacture of vascular grafts. The ePTFE-based BeGraft $^{\mathrm{TM}}$ coronary stent graft system (Bentley Innomed $\mathrm{GmbH}$, Hechingen, Germany) combines a single layer cobalt-chromium stent platform with an ePTFE membrane clamped at the stent ends [12]; whereas, polyurethanes are known for their excellent biocompatibility in combination with their well-known physiochemical and mechanical properties [12]. They are commonly used in cardiovascular applications, such as heart valves and pacemaker leads. Polyurethane-covered stents have been in use for the peripheral vasculature for decades. The polyurethane-based PK Papyrus ${ }^{\text {TM }}$ coronary stent system (Biotronik, Bülach, Switzerland) combines a stent platform, covered by a $90 \mu \mathrm{m}$ thick polymer using electrospinning technology that yields a thinner and relatively flexible material [12]. The detailed characteristics of both devices are shown in Table 1.

To cover those CS devices, we used third-generation DES, preferably cobalt-chromium based sirolimus- or everolimus-eluting coronary stent systems (Orsiro ${ }^{\mathrm{TM}}$ or Xience Alpine/ Sierra ${ }^{\mathrm{TM}}$ DES devices, respectively). Notably, the DES was longer than the covered stent, which meant that the covered stent was completely "buried" at both ends (overhanging the edges). Post-dilatation was performed in all patients using non-compliant balloons 
Table 1. Product characteristics of the used covered stent devices [12].

\begin{tabular}{lcc}
\hline & $\begin{array}{c}\text { BeGraft } \\
\text { (Bentley Innomed) }\end{array}$ & $\begin{array}{c}\text { PK Papyrus }^{\mathrm{TM}} \\
\text { (Biotronik) }\end{array}$ \\
\hline $\begin{array}{l}\text { Graft material } \\
\text { Stent material/design }\end{array}$ & $\begin{array}{c}\text { ePTFE } \\
\text { CoCr (L-605) } \\
\text { Single layer }\end{array}$ & $\begin{array}{c}\text { Electrospun polyurethane } \\
\text { CoCr (L-605) with silicon carbide coating } \\
\text { Single layer }\end{array}$ \\
Guide catheter compatibility & $5 \mathrm{Fr}$ & $5 \mathrm{Fr}$ (stents $<4.0 \mathrm{~mm})$ \\
Crimped profile [mm] & & $6 \mathrm{Fr}($ stents $\geq 4.0 \mathrm{~mm})$ \\
Stent diameters [mm] & $1.1-1.4$ & $1.18-1.55$ \\
Stent lengths [mm] & $2.5-5.0$ & $2.5-5.0$ \\
Nominal implantation & $8-24$ & $15-26$ \\
pressure [atm] & $11 \mathrm{~atm}(2.5-4.0 \mathrm{~mm})$ & 8 atm $(2.5-3.5 \mathrm{~mm})$ \\
\hline
\end{tabular}

Information obtained from product catalogues and Kilic et al. [12]; atm — atmospheres; $\mathrm{CoCr}$ - cobalt-chromium; ePTFE — expanded polytetrafluoroethylene; $\mathrm{Fr}$ - French

in order to achieve optimal stent expansion and apposition. For post-dilatation, the balloon-diameter is usually selected either on visual estimation (if possible, applying a balloon-to-vessel ratio of $1: 1)$ or based upon optical coherence tomography (OCT) measurement at our site. In selected cases with underlying heavy calcifications resulting in residual stent under-expansion, we might choose an $\mathrm{OPN}^{\mathrm{TM}}$ non-compliant balloon at very high pressure (30$-50 \mathrm{~atm}$ ) using a diameter $0.5 \mathrm{~mm}$ smaller than the estimated/measured vessel size.

\section{Data collection and outcome definitions}

Information about the patient characteristics, including previous medical history and medication, vital parameters, laboratory values, $\mathrm{PCI}$ indication, as well as periprocedural findings/management were collected by chart review. Lesion characteristics were classified according to the American College of Cardiology (ACC) and American Heart Association (AHA) lesion classification [13]. Coronary calcifications were identified as easily apparent radiopacities within the vascular wall at the site of the lesions and were classified as none/ /mild, moderate (radiopacities noted only during the cardiac cycle before contrast injection), and severe (radiopacities observed without cardiac motion prior to contrast injection commonly compromising both sides of the arterial lumen) [14]. Chronic total occlusions were defined as a more than three-month-old obstruction of native coronary arteries, free of any luminal continuity, and thrombolysis in myocardial infarction (TIMI) flow 0.

Coronary perforations were graded according to the Ellis Classification: Type I, Extraluminal crater without extravasation; Type II, Pericardial or myocardial blush without contrast jet extravasation; Type III, Extravasation through frank (>1 mm) perforation; and Type III, cavity spilling, cavity perforation into an anatomic cavity chamber, spilling coronary sinus, etc. [15].

Our clinical endpoints of interest included death from any cause, cardiac death, target vessel revascularization (TVR) myocardial infarction (MI), clinically driven TLR, and ST [16]. Cardiac death and clinically driven TLR were defined as suggested by the Academic Research Consortium (ARC) criteria [17, 18]. For MI, we applied the universal definition [16]. ST was classified as definite, probable, and possible $[17,18]$.

Follow-up information was collected by chart review and from visits to our clinics. All coronary angiograms and OCT runs were reviewed and adjudicated by M.B. and F.C.

\section{OCT acquisition and analysis}

In the case of TVR, our internal protocol suggested an OCT if possible. OCT investigations were acquired before and optimally after PCI. For OCT, we used the Optis Ilumien ${ }^{\mathrm{TM}}$ system and the Dragon Fly Duo OCT Imaging Catheter (St. Jude Medical/Abbott Vascular, Santa Clara, CA) with motorized pullback $(25 \mathrm{~mm} / \mathrm{s})$ using a non-occlusive flushing technique according to the manufacturer's recommendations. Images of the $\mathrm{CS}$ and of the reference segments $10 \mathrm{~mm}$ proximal and distal of the previously implanted stent devices were acquired. OCT pullbacks were registered and assessed offline using dedicated software (OPTIS ${ }^{\mathrm{TM}}$ Imaging Software, Abbott Vascular, Santa Clara, CA). We 
applied the same methodology and definitions as described elsewhere earlier [18].

\section{Statistical methodology}

Categorical variables are displayed as absolute numbers and percentages, and continuous variables are presented as means ( \pm standard deviations) or medians (interquartile ranges [IQR]), as appropriate.

\section{Results}

A total of 23 consecutive patients were included in this registry over a period of 5 years (2015-2020). Baseline characteristics of the study population are depicted in Table 2 . In brief, the mean age was $67 \pm 14$ years, and most of the patients were male $(74 \%)$. Half (52\%) of the patients presented with a stable angina equivalent (chronic coronary syndrome), while the remaining had an acute coronary syndrome.

\section{Procedural characteristics}

The indication for the use of CS, lesion characteristics, and details of the procedures are summarized in Table 3. The reasons for implantation of CS included treatment of coronary aneurysms, coronary perforations, and deep dissections at the ostium of the right coronary artery among $7(30 \%)$ patients, $13(57 \%)$ patients, and 3 (13\%) patients, respectively. Two illustrative case vignettes are highlighted in Figure 1 and 2.

In total, 24 CS devices were deployed: 13 (54\%) Papyrus ${ }^{\mathrm{TM}}$ and 11 (46\%) BeGraft ${ }^{\mathrm{TM}}$ devices. The mean size and length of the CS devices were $3.6 \pm 0.6 \mathrm{~mm}$ and $19.1 \pm 3.8 \mathrm{~mm}$, respectively. The CS were covered with a total of 52 DES with a mean size and length of $3.6 \pm 0.5 \mathrm{~mm}$ and $30.2 \pm 7.7 \mathrm{~mm}$. All stents were post-dilated using adequately sized non-compliant balloons (mean balloon size $3.9 \pm$ $\pm 0.4 \mathrm{~mm}$, mean inflation pressure $19 \pm 6 \mathrm{~atm}$ ).

In $12(52 \%)$ patients, the coronary CS was directly deployed at the site of native lesion and then covered using a DES. All patients undergoing treatment of a coronary aneurysm $(n=7)$ and 5 patients with coronary perforations (Ellis type $2-3$ ) after lesion preparation were treated by applying the following sequence: $\mathrm{CS} \rightarrow \mathrm{DES}$. Among 11 patients, coronary perforation/rupture occurred after DES implantation (particularly after postdilatation). In those scenarios, the CS was used to seal the perforation site and stop the bleeding. The DES was implanted to finally cover the CS (sequence: DES $\rightarrow$ CS $\rightarrow$ DES).
Table 2. Baseline characteristics of the study population $(n=23)$.

\begin{tabular}{|c|c|}
\hline Age [years] & $67 \pm 14$ \\
\hline Female sex & $6 / 23(26 \%)$ \\
\hline \multicolumn{2}{|l|}{ Cardiovascular risk factors: } \\
\hline Arterial hypertension & $18 / 23(78 \%)$ \\
\hline Smoking & $11 / 23(48 \%)$ \\
\hline Diabetes & $4 / 23(17 \%)$ \\
\hline Dyslipidemia & $16 / 23(70 \%)$ \\
\hline \multicolumn{2}{|l|}{ Previous medical history: } \\
\hline Previous myocardial infarction & $9 / 23(39 \%)$ \\
\hline Previous $\mathrm{PCl}$ & $8 / 23(35 \%)$ \\
\hline Previous CABG & $1 / 23(4 \%)$ \\
\hline Established renal failure* & $7 / 23(30 \%)$ \\
\hline Left ventricular ejection fraction [\%] & $53 \pm 7$ \\
\hline \multicolumn{2}{|l|}{ Clinical presentation: } \\
\hline Stable angina equivalent (CCS) & $12 / 23(52 \%)$ \\
\hline Unstable angina/NSTEMI & $9 / 23(39 \%)$ \\
\hline STEMI & $2 / 23(9 \%)$ \\
\hline \multicolumn{2}{|l|}{ Access management: } \\
\hline Radial approach & $15 / 23(65 \%)$ \\
\hline Femoral approach & $8 / 23(35 \%)$ \\
\hline \multicolumn{2}{|l|}{ Antithrombotic management $(\%)^{* *}$ : } \\
\hline Heparin & $23 / 23(100 \%)$ \\
\hline Eptifibatide & $5 / 23(22 \%)$ \\
\hline ASA & $23 / 23(100 \%)$ \\
\hline Clopidogrel & $7 / 23(30 \%)$ \\
\hline Ticagrelor & $13 / 23(57 \%)$ \\
\hline Prasugrel & $3 / 23(13 \%)$ \\
\hline Rivaroxaban & $4 / 23(17 \%)$ \\
\hline
\end{tabular}

*Defined as estimated glomerular filtration $<45 \mathrm{~mL} / \mathrm{min} / 1.73 \mathrm{~m}^{2}$ (CKD-EPI); **Including post-procedural antithrombotic management; ASA - acetylsalicylic acid; CABG — coronary artery by-pass grafting; CCS - chronic coronary syndrome; NSTEMI non-ST segment elevation myocardial infarction; $\mathrm{PCl}-$ percutaneous coronary intervention; STEMI - ST-segment elevation myocardial infarction

\section{Outcomes}

The median time of follow-up was 24.5 (IQR 11.7-37.9) months. Short- and long-term outcomes are summarized in Table 4 . No patients experienced probable or definite ST. Only 1 patient required TLR due to clinically significant ISR 6 months after CS implantation. In this case, a coronary perforation had occurred after treatment of a highly calcified ostial RCA lesion (the treatment sequence at the index procedure was DES $\rightarrow \mathrm{CS} \rightarrow \mathrm{DES})$. In this specific case, we identified recoil in combination with neo-intima formation as the mechanisms leading to ISR. Consequently, we 
Table 3. Procedural details $(n=23)$.

\begin{tabular}{|c|c|}
\hline \multicolumn{2}{|l|}{ Vessels treated: } \\
\hline LM & $1 / 23(4 \%)$ \\
\hline LAD & $7 / 23(30 \%)$ \\
\hline LCX & $0 / 23(0 \%)$ \\
\hline $\mathrm{RCA}$ & $15 / 23(66 \%)$ \\
\hline \multicolumn{2}{|l|}{ Coronary calcification: } \\
\hline No calcification & $4 / 23(17 \%)$ \\
\hline Mild calcification & $2 / 23(9 \%)$ \\
\hline Moderate calcification & $6 / 23(26 \%)$ \\
\hline Severe calcification & $11 / 23(48 \%)$ \\
\hline Chronic total occlusion & $5 / 23(22 \%)$ \\
\hline \multicolumn{2}{|l|}{ Indication for covered stent use: } \\
\hline Coronary aneurysm & $7 / 23(30 \%)$ \\
\hline Coronary perforation & $13 / 23(57 \%)$ \\
\hline Type 2 perforation & $9 / 13(69 \%)$ \\
\hline Type 3 perforation & $4 / 13(31 \%)$ \\
\hline Aorto-ostial dissection & $3 / 23(13 \%)$ \\
\hline Pericardial tamponade & $2 / 23(9 \%)$ \\
\hline \multicolumn{2}{|l|}{ Covered stent devices: } \\
\hline No. of devices used & 24 \\
\hline Papyrus $^{\mathrm{TM}}$ & $13 / 23(55 \%)$ \\
\hline BeGraft $^{\mathrm{TM}}$ & $10 / 23(44 \%)$ \\
\hline Size $[\mathrm{mm}]$ & $3.6 \pm 0.6$ \\
\hline Length [mm] & $19.1 \pm 3.8$ \\
\hline Implantation pressure [atm] & $13 \pm 2$ \\
\hline \multicolumn{2}{|l|}{ Drug-eluting stent devices: } \\
\hline No. of devices used & 52 \\
\hline Xience $^{\mathrm{TM}}$ EES & $51 \%$ \\
\hline Orsiro $^{\mathrm{TM}}$ SES & $44 \%$ \\
\hline Other DES & $5 \%$ \\
\hline Size $[\mathrm{mm}]$ & $3.6 \pm 0.5$ \\
\hline Length $[\mathrm{mm}]$ & $30.2 \pm 7.7$ \\
\hline Post-dilatation balloon size [mm] & $3.9 \pm 0.4$ \\
\hline Post-dilatation pressure [atm] & $19 \pm 6$ \\
\hline \multicolumn{2}{|c|}{ Combination of covered stent and DES: } \\
\hline 3 layers (DES/covered stent/DES) & $11 / 23(48 \%)$ \\
\hline 2 layers (covered stent/DES) & $12 / 23(52 \%)$ \\
\hline
\end{tabular}

DES - drug-eluting stents; EES - everolimus-eluting stent; No. — number; LAD - left anterior descending artery; LCX — left circumflex; LM — left main; RCA — right coronary artery; SES sirolimus-eluting stent

treated this with aggressive post-dilatation (using an $\mathrm{OPN}^{\mathrm{TM}}$ non-compliant balloon at high-pressure, $>30 \mathrm{~atm}$ ) and implantation of another DES (Xience ${ }^{\mathrm{TM}}$ $4.0 \times 18 \mathrm{~mm}$ ), also see Supplemental Figure 1 . A total of 5 patients had TVR, and 1 patient died 30 days after PCI due to non-cardiovascular causes.

\section{Discussion}

We analyzed outcomes of a cohort of patients requiring CS implantation for treatment of coronary aneurysms, perforations, or dissections and who were managed with a novel hybrid approach aiming to bury the CS behind a contemporary DES. This strategy appears to be safe and associated with excellent short- and long-term results in this registry, including a very low risk for TLR.

Coronary CS often comprises life-saving bailout devices in the setting of significant coronary artery perforation, in order to seal the perforated site. However, one needs to take into account the fact that residual endoleaks remain a concern and may ultimately lead to pericardial effusion and tamponade $[3,19]$. This may even be encountered with some delay $[3,19]$. Of note, previously published series including patients who experienced coronary perforations and who were treated with the implantation of a CS have demonstrated that a second or a third device is required in up to $20 \%$ cases to cover those endoleaks [20,21]. In this context, the main mechanisms responsible for CS failure probably reflect the difficulty in achieving complete apposition - even with adequate post-dilatation and/or the lack of radial strength [19]. In our series 2 out of 13 patients with coronary perforation (15\%) had a pericardial tamponade, requiring pericardiocentesis. In both patients, the initial strategy was to treat the coronary perforation with the implantation of CS only, but the presence of tamponade led to a change of strategy and the additional implantation of a DES to close the endoleak. Interestingly, in both cases endoleaks were barely visible on coronary angiography. Our approach of covering the CS completely with a DES addresses some of the aforementioned mechanical challenges and enhances stent apposition and reduces the chance of an endoleak requiring additional CS to seal a coronary perforation, which is also highlighted in Figure 3.

Compared to previous studies, which indicated high rates of TLF and TVR (5-25\%) during short- and long-term follow-up (> 12 months), our results implicate that better outcomes (TLF rate $<5 \%$ ) might be achieved after implantation of CS devices by applying the presented hybrid approach $[3,6,7,22-24]$.

Our data should be interpreted cautiously and considered as hypothesis-generating. Considering possible explanations for the rather favorable outcomes that we observed in our cohort of patients 


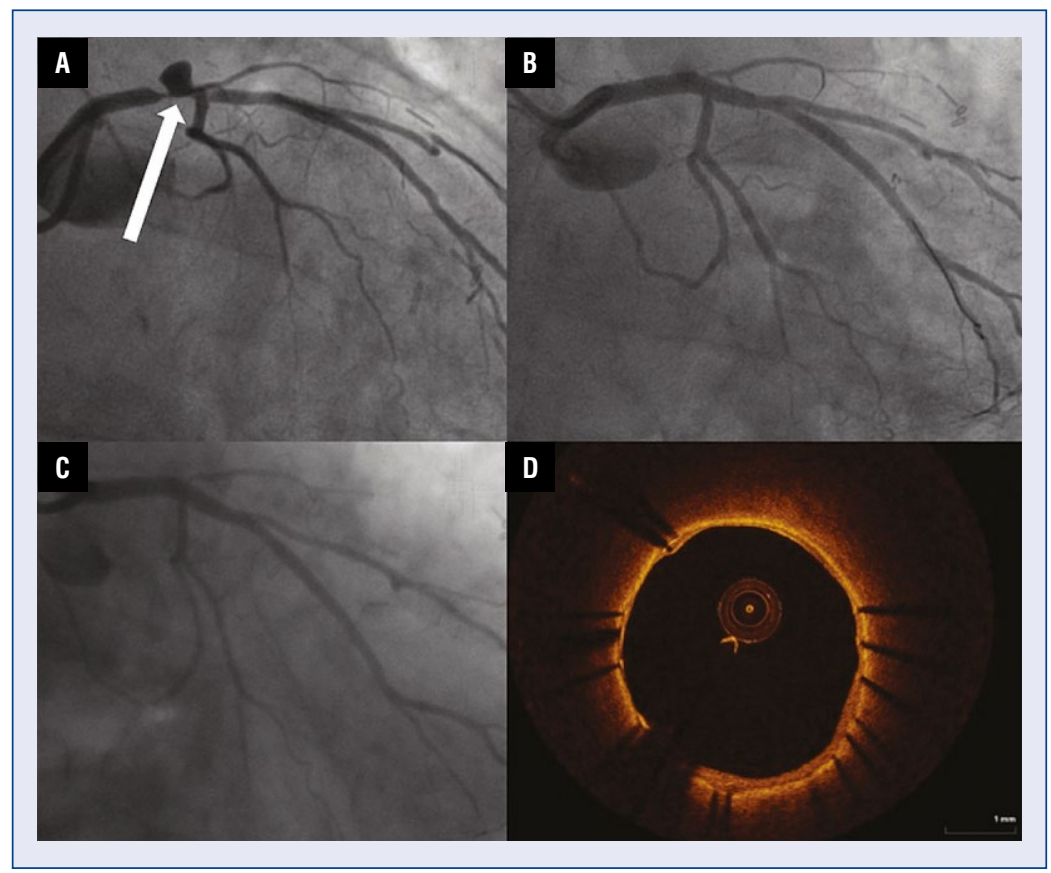

Figure 1. Treatment of a coronary aneurysm using a combination of a covered and drug-eluting stent. A. This panel demonstrates a significant left main stenosis $(90 \%)$ followed by a large aneurysm. The aneurysm was initially sealed with a covered stent (Papyrus ${ }^{\mathrm{TM}} 3.5 \times 15 \mathrm{~mm}$ ). A drug-eluting stent (Xience ${ }^{\mathrm{TM}} 3.5 \times 28 \mathrm{~mm}$ ) was then implanted over the covered stent and post-dilated with a $4.0 \mathrm{~mm}$ non-compliant balloon at 18 atm achieving a good result (B). Followup coronary angiography was performed 6 months later $(\mathbf{C})$ showing a complete exclusion of the aneurysm and no in-stent restenosis. D. This was confirmed by optical coherence tomography demonstrating good healing, including coverage of all struts and only minimal neointimal hyperplasia.

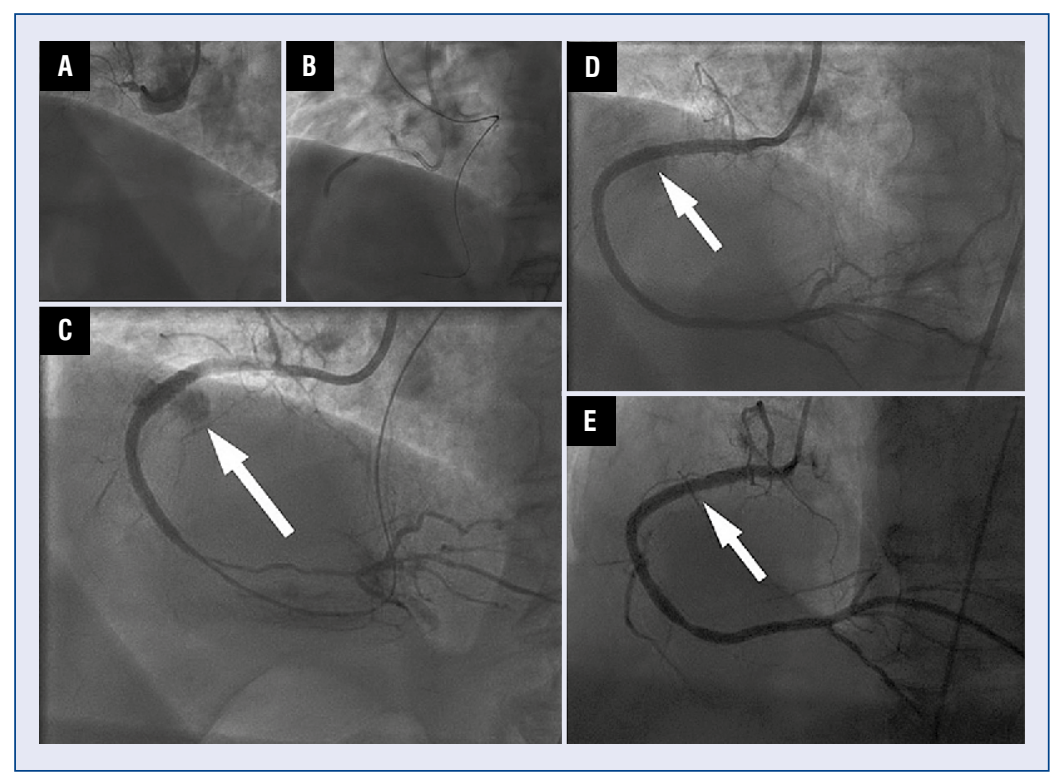

Figure 2. Sealing of a coronary perforation using a hybrid approach combining covered and drug-eluting stents (DES). A. A chronic total occlusion of the right coronary artery (RCA). Retrograde recanalization was performed, (B) but after a DES (Xience ${ }^{\mathrm{TM}} 3.5 \times 28 \mathrm{~mm}$ ) was implanted, a large coronary perforation was observed (Ellis type III) (C). Balloon occlusion did not stop the bleeding, and the perforation was consecutively sealed with a Papyrus $3.5 \times 20 \mathrm{~mm}$ covered stent. The covered stent was then buried using a Xience ${ }^{\mathrm{TM}} 3.5 \times 38 \mathrm{~mm}$ DES, which led to an angiographically good result (D). Surveillance coronary angiography was performed 6 months later (E) confirming the good result in the proximal RCA. 
Table 4. Short- and long-term outcomes.

\begin{tabular}{lcc}
\hline & $\mathbf{1}$ month $(\mathbf{n}=\mathbf{2 3})$ & $\mathbf{1 2}$ months $(\mathbf{n}=\mathbf{1 9})$ \\
\hline Myocardial infarction & $1 / 23(4 \%)$ & $1 / 19(5 \%)$ \\
Target vessel revascularization & $1 / 23(4 \%)$ & $1 / 19(5 \%)$ \\
Target lesion revascularization & $0 / 23(0 \%)$ & $0 / 19(0 \%)$ \\
Probable or definite stent thrombosis & $0 / 23(0 \%)$ & $0 / 19(0 \%)$ \\
Death: & & $1 / 19(5 \%)$ \\
Cardiovascular cause & $0 / 23(0 \%)$ & $1 / 23(4 \%)$ \\
Other cause & $1 / 26 \%)$ \\
\hline
\end{tabular}

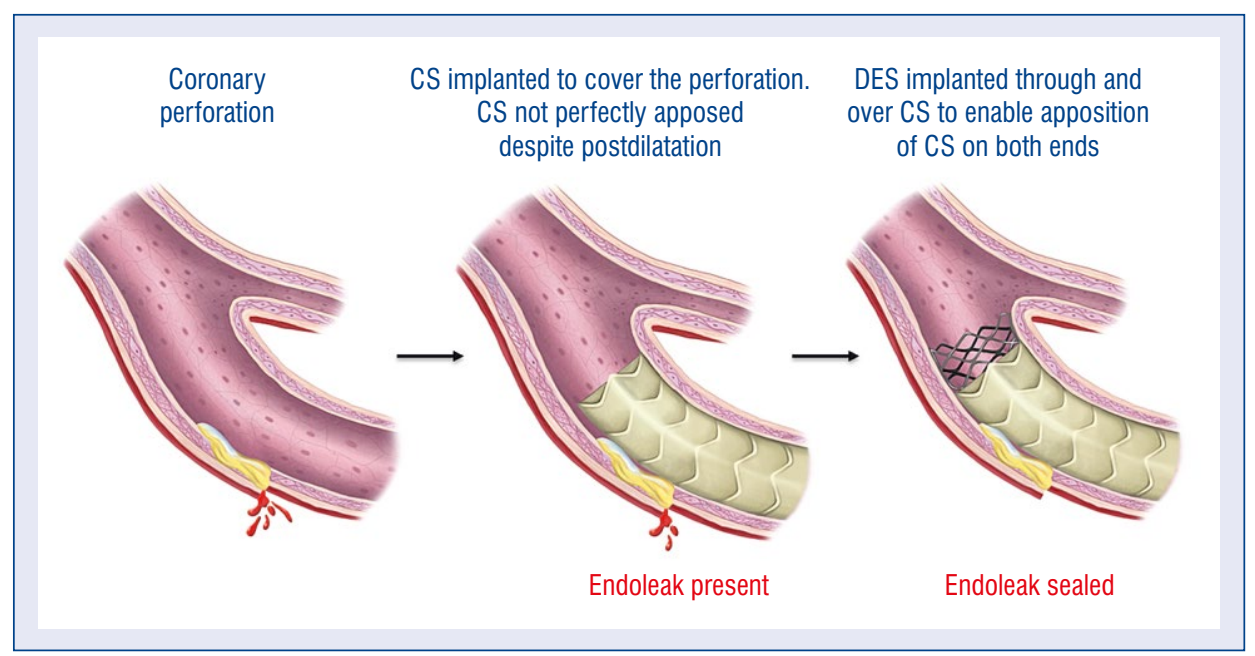

Figure 3. Elimination of an endoleak after implantation of a covered stent (CS) by overstenting ("burying") it with a drug-eluting stent (DES).

treated with $\mathrm{CS}$, we think that the following three mechanisms may play an important role: (I) although we did not routinely perform intravascular imaging to guide implantation, it is plausible that the additional implantation of a DES leads to a larger final lumen; (II) the presence of 2 layers of stents might provide enhanced radial strength, especially in fibro-calcific lesions, and therefore improved stent expansion; (III) additionally, a good stent expansion and apposition leads to enhanced laminar flow, which may limit the development of neoatherosclerosis and lower the risk for acute and subacute ST.

As mentioned before, the healing response after implantation of a prosthetic graft is complicated and involves a complex interplay between pro-thrombotic processes, and growth of extracellular and cellular matrix [8]. Additionally, acute vessel injury leads by itself to a healing response with neointimal hyperplasia [11]. The presence of a DES seems to positively affect these processes - vascular healing was excellent in our patients, as demonstrated in Figures 1 and 2. Interestingly, even in patients treated with 3 layers of stent (DES-CS-DES) we did not observe a signal of ISR or ST, which indicates that the luminal presence of DES might contribute to a more controlled vascular healing response.

\section{Limitations of the study}

We understand these results have some limitations and should be interpreted cautiously because they derive from a single center registry study. Also, we lack a comparison group. Therefore, more prospective and follow-up data are required to confirm our results. Also, it remains uncertain if our approach also applies to other coronary CS devices, because we only used polytetrafluoroethylene and polyurethanes containing devices.

\section{Conclusions}

This study suggests that rates of ST and ISR may be improved if CSs are overstented ("buried") under contemporary DESs. If confirmed in fur- 
ther studies, this approach could help to improve short- and long-term outcomes of coronary CS implantation.

Conflict of interest: Matthias Bossard has received consulting and speaker fees from Amgen, AstraZeneca, Bayer, and Mundipharma. Richard Kobza has received institutional grants from Abbott, Biosense-Webster, Boston-Scientific, Biotronik, Medtronic, and SisMedical. Florim Cuculi has received consulting and speaker fees from SIS Medical and Abbott Vascular. Giacomo Maria Cioffi, Mustafa Yildirim, Federico Moccetti, Mathias Wolfrum, Adrian Attinger, and Stefan Toggweiler report no conflicts of interest.

\section{References}

1. Xenogiannis I, Brilakis E. Advances in the treatment of coronary perforations. Catheter Cardiovasc Interv. 2019; 93(5): 921-922, doi: 10.1002/ccd.28205.

2. Kawsara A, Núñez Gil IJ, Alqahtani F, et al. Management of Coronary Artery Aneurysms. JACC Cardiovasc Interv. 2018; 11(13): 1211-1223, doi: 10.1016/j.jcin.2018.02.041, indexed in Pubmed: 29976357.

3. Copeland KA, Hopkins JT, Weintraub WS, et al. Long-term follow-up of polytetrafluoroethylene-covered stents implanted during percutaneous coronary intervention for management of acute coronary perforation. Catheter Cardiovasc Interv. 2012; 80(1): 53-57, doi: 10.1002/ccd.23339, indexed in Pubmed: 21953680.

4. Nagaraja V, Schwarz K, Moss S, et al. Outcomes of patients who undergo percutaneous coronary intervention with covered stents for coronary perforation: A systematic review and pooled analysis of data. Catheter Cardiovasc Interv. 2020; 96(7): 1360-1366, doi: 10.1002/ccd.28646, indexed in Pubmed: 31850685.

5. Kandzari DE, Birkemeyer R. PK Papyrus covered stent: Device description and early experience for the treatment of coronary artery perforations. Catheter Cardiovasc Interv. 2019; 94(4): 564-568, doi: 10.1002/ccd.28306, indexed in Pubmed: 31033148.

6. Kufner S, Schacher N, Ferenc M, et al. Outcome after new generation single-layer polytetrafluoroethylene-covered stent implantation for the treatment of coronary artery perforation. Catheter Cardiovasc Interv. 2019; 93(5): 912-920, doi: 10.1002/ ccd.27979, indexed in Pubmed: 30467994.

7. Jurado-Román A, Rodríguez O, Amat I, et al. Clinical outcomes after implantation of polyurethane-covered cobalt-chromium stents. Insights from the Papyrus-Spain registry. Cardiovasc Revasc Med. 2020 [Epub ahead of print], doi: 10.1016/j.carrev.2020.08.017, indexed in Pubmed: 32859538.

8. Ravi S, Chaikof EL. Biomaterials for vascular tissue engineering. Regen Med. 2010; 5(1): 107-120, doi: 10.2217/rme.09.77, indexed in Pubmed: 20017698.

9. Goh ET, Wong E, Farhatnia Y, et al. Accelerating in situ endothelialisation of cardiovascular bypass grafts. Int J Mol Sci. 2014; 16(1): 597-627, doi: 10.3390/ijms16010597, indexed in Pubmed: 25551605.

10. Stefanini GG, Holmes DR. Drug-eluting coronary-artery stents. N Engl J Med. 2013; 368(3): 254-265, doi: 10.1056/NEJMra1210816, indexed in Pubmed: 23323902.
11. Farb A, Sangiorgi G, Carter AJ, et al. Pathology of acute and chronic coronary stenting in humans. Circulation. 1999; 99(1): 44-52, doi: 10.1161/01.cir.99.1.44, indexed in Pubmed: 9884378.

12. Kilic ID, Fabris E, Serdoz R, et al. Coronary covered stents. EuroIntervention. 2016; 12(10): 1288-1295, doi: 10.4244/EIJV12I10A210, indexed in Pubmed: 27866138.

13. Ryan TJ, Bauman WB, Kennedy JW, et al. Guidelines for percutaneous transluminal coronary angioplasty. A report of the American Heart Association/American College of Cardiology Task Force on Assessment of Diagnostic and Therapeutic Cardiovascular Procedures (Committee on Percutaneous Transluminal Coronary Angioplasty). Circulation. 1993; 88(6): 2987-3007, doi: 10.1161/01.cir.88.6.2987, indexed in Pubmed: 8252713.

14. Mintz GS, Popma JJ, Pichard AD, et al. Patterns of calcification in coronary artery disease. A statistical analysis of intravascular ultrasound and coronary angiography in 1155 lesions. Circulation. 1995; 91(7): 1959-1965, doi: 10.1161/01.cir.91.7.1959, indexed in Pubmed: 7895353.

15. Ellis SG, Ajluni S, Arnold AZ, et al. Increased coronary perforation in the new device era. Incidence, classification, management, and outcome. Circulation. 1994; 90(6): 2725-2730, doi: 10.1161/01.cir.90.6.2725, indexed in Pubmed: 7994814.

16. Thygesen K, Alpert J, Jaffe A, et al. Fourth Universal Definition of Myocardial Infarction (2018). Circulation. 2018; 138(20): e618-e651, doi: 10.1161/cir.0000000000000617.

17. Cutlip DE, Windecker S, Mehran R, et al. Academic Research Consortium. Clinical end points in coronary stent trials: a case for standardized definitions. Circulation. 2007; 115(17): 2344 2351, doi: 10.1161/CIRCULATIONAHA.106.685313, indexed in Pubmed: 17470709 .

18. Cuculi F, Puricel S, Jamshidi P, et al. Optical coherence tomography findings in bioresorbable vascular scaffolds thrombosis. Circ Cardiovasc Interv. 2015; 8(10): e002518, doi: 10.1161/CIRCINTERVENTIONS.114.002518, indexed in Pubmed: 26399265.

19. von Stempel C, Fayed H, Goode JA, et al. Viabahn stent graft in the management of a grade 3 coronary perforation. CVIR Endovasc. 2019 ; 2(1): 6 , doi: 10.1186/s42155-019-0050-8, indexed in Pubmed: 32026215.

20. Ly H, Awaida JPS, Lespérance J, et al. Angiographic and clinical outcomes of polytetrafluoroethylene-covered stent use in significant coronary perforations. Am J Cardiol. 2005; 95(2): 244-246, doi: 10.1016/j.amjcard.2004.09.010, indexed in Pubmed: 15642559.

21. Lansky AJ, Yang Ym, Khan Y, et al. Treatment of coronary artery perforations complicating percutaneous coronary intervention with a polytetrafluoroethylene-covered stent graft. Am J Cardiol. 2006; 98(3): 370-374, doi: 10.1016/j.amjcard.2006.02.041, indexed in Pubmed: 16860026.

22. Wang HJ, Lin JJ, Lo WY, et al. Clinical outcomes of polytetrafluoroethylene-covered stents for coronary artery perforation in elderly patients undergoing percutaneous coronary interventions. Acta Cardiol Sin. 2017; 33(6): 605-613, doi: 10.6515/ ACS20170625A, indexed in Pubmed: 29167613.

23. Pavani M, Cerrato E, Latib A, et al. Acute and long-term outcomes after polytetrafluoroethylene or pericardium covered stenting for grade 3 coronary artery perforations: Insights from G3-CAP registry. Catheter Cardiovasc Interv. 2018; 92(7): 1247-1255, doi: 10.1002/ccd.27789, indexed in Pubmed: 30244520.

24. Rosseel L, Scott B, Prihadi E, et al. Is a covered stent justifiable in the treatment of coronary artery perforation? An observational analysis of long-term results of two different covered stent types. Catheter Cardiovasc Interv. 2019; 93(3): 419-425, doi: 10.1002/ ccd.27892, indexed in Pubmed: 30280487. 